\title{
TORCH - an Innovative High-Precision Time-of-Flight PID Detector for the LHCb Upgrade
}

\author{
Klaus Föhl, Nick Brook, Lucía Castillo García, Euan N. Cowie, David Cussans, Roger Forty, Christoph Frei, \\ Rui Gao, Thierry Gys, Neville Harnew, Didier Piedigrossi, Maarten van Dijk
}

\begin{abstract}
TORCH is a Cherenkov time-of-flight detector being developed as a particle identification system for the upgraded LHCb experiment. The DIRC-type detector is located at $10 \mathrm{~m}$ distance from the interaction point with an area of $30 \mathrm{~m}^{2}$ and is formed from $10 \mathrm{~mm}$ thick synthetic amorphous fused silica plates. In this Cherenkov radiator the photons that propagate by total internal reflection to the plate edge are focussed onto an array of position-sensitive micro-channel plate sensors. Combining the photon timings the goal is to achieve a $\sigma=15 \mathrm{ps}$ timing resolution per particle, yielding $3 \sigma$ pion-kaon separation up to $10 \mathrm{GeV} / \mathrm{c}$ or better. Requirements for the photon detectors are presented together with preliminary prototype results. Preparations for the upcoming test beam are discussed. Optical design studies have been performed for using one of the available bar boxes of the BaBar DIRC detector containing assembled quartz radiator bars in a future test beam experiment.
\end{abstract}

\section{INTRODUCTION}

$\mathbf{T}$ HE LHCb experiment [1] is a forward spectrometer (Fig. 1) optimised for the study of heavy-flavour hadron production. The experiment will measure the CKM parameters of the Unitarity Triangle with high precision and search for physics beyond the Standard Model. The LHCb Upgrade [2] is designed for an increase of an order of magnitude in luminosity, and particle identification (PID) is vital for the experiment.

The TORCH (Time Of internally Reflected CHerenkov light) detector [3], [4], [5] is an innovative high-precision timeof-flight system for $\pi$-K PID incorporating DIRC (Detection of Internally Reflected Cherenkov light) [6] methods. As the design is suitable for covering large areas, up to tens of square metres, it can be located in a gap downstream of the T1T3 tracking stations and upstream of RICH2 (Fig. 1). The TORCH detector will cover the momentum range from 1 to $10 \mathrm{GeV} / \mathrm{c}$. The timing information is made up of two parts, see Fig. 2. The first part is the particle time-of-flight over a flight path of $10 \mathrm{~m}$ from the $\mathrm{LHCb}$ interaction point (IP) to the radiator plate. The second part is the Cherenkov photons' time-of-propagation in the radiator plate. The photon arrival time is measured by micro-channel plate (MCP) sensors.

Klaus Föhl, Lucía Castillo García, Roger Forty, Christoph Frei, Thierry Gys and Didier Piedigrossi are with CERN, PH Department, CH-1211 Geneva 23, Switzerland; email:klaus.foehl@cern.ch

Luc ia Castillo García is also with the Laboratory for High Energy Physics, Ecole Polytechnique Fédérale de Lausanne, CH-1015 Lausanne, Switzerland

Nick Brook, Euan N. Cowie and David Cussans are with H. H. Wills Physics Laboratory, University of Bristol, Bristol, BS8 1TL, United Kingdom

Rui Gao and Neville Harnew are with Department of Physics, University of Oxford, Oxford, OXI 3RH, United Kingdom

Manuscript received November 30, 2014

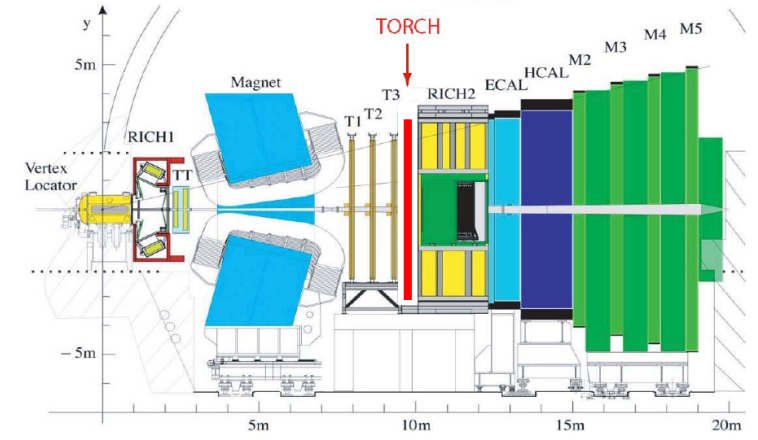

Fig. 1. Side view of $\mathrm{LHCb}$ with the proposed position of TORCH between the tracking stations T1-T3 to the left and $\mathrm{RICH} 2$ to the right.

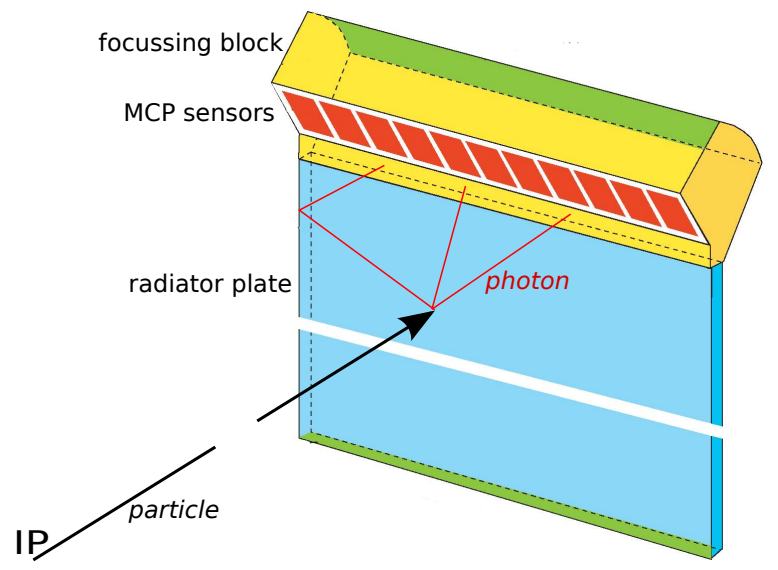

Fig. 2. TORCH principle with the particle path from the IP to the radiator shown in black and three sample photon propagation paths shown in red.

The colliding-beam $\mathrm{LHCb}$ experiment has a forward spectrometer geometry covering $\pm 10-300$ mrad horizontally and $\pm 10-250 \mathrm{mrad}$ vertically relative to the beam axis. At $10 \mathrm{~m}$ distance from the IP a vertical structure of $6 \mathrm{~m}$ width and $5 \mathrm{~m}$ height is thus required. In the baseline design [2] TORCH consists of $2 \times 9$ modules of synthetic amorphous fused silica (quartz) radiator plates, each $10 \mathrm{~mm}$ thick and $2.5 \mathrm{~m} \times 0.66 \mathrm{~m}$ in size. These are equipped on one end, either at the top or at the bottom, with a focussing block (see Fig. 3) with a mirrored cylindrical surface and instrumented with an array of pixelated MCP sensors to detect single photons.

Cherenkov photons are emitted by charged particles passing through the quartz radiator. These photons propagate by total internal reflection to the edge of the quartz plate. The focussing 


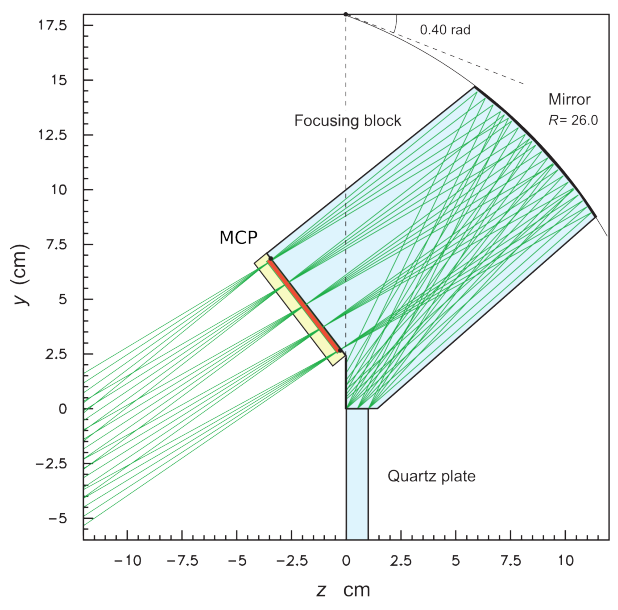

Fig. 3. Side view of the TORCH focussing block geometry. Taking the MCP pixelisation into account, one can measure photon angles with an error of $\sigma=1 \mathrm{mrad}$.

block converts one component of the photon angle, the component lying in the image plane of Fig. 3, into a position on the focal plane. The second, lateral angle component is derived from the geometry of the particle trajectory through the quartz and the lateral photon position on the sensor.

\section{REQUIRED PERFORMANCE}

The TORCH detector measures both the position and the arrival time of the imaged photons. Figure 4 shows an example of photon arrival time difference when comparing kaons to pions. The case of a $9.5 \mathrm{~m}$ flight path normal to the radiator and a $2 \mathrm{~m}$ vertical photon propagation distance is shown, and both kaons and pions happen to emit a photon at the same angle of $822 \mathrm{mrad}$. For the case of $10 \mathrm{GeV} / \mathrm{c}$ where kaon and pion photons have wavelengths of $394 \mathrm{~nm}$ and $410 \mathrm{~nm}$ and propagate with speeds of $\mathrm{c} / 1.517$ and $\mathrm{c} / 1.512$, respectively, one finds a 35 ps time-of-flight difference and a 49 ps time-ofpropagation difference for the overall $84 \mathrm{ps}$ time difference. However, when the particle trajectory passes closer to the readout face the photon propagation distance can be much smaller and the time-of-propagation difference shrinks. Hence one has to base the stipulated $3 \sigma$ separation mostly on the time-of-flight difference, and has defined the goal to achieve a timing resolution of $\sigma=15$ ps per particle.

Figure 5 gives the calculated PID performance of the Cherenkov detectors in LHCb. RICH1 and RICH2 with their gas radiators, $\mathrm{C}_{4} \mathrm{~F}_{10}$ and $\mathrm{CF}_{4}$, respectively, provide the highermomentum PID. The lower-momentum range of $<10 \mathrm{GeV} / \mathrm{c}$ was previously covered by aerogel in the RICH1 system. However the aerogel in RICH1 is less effective at higher luminosity due to its low photon yield and increased background, and has been removed for the $\mathrm{LHCb}$ upgrade [7], and TORCH has been proposed to replace the lost momentum coverage.

Small-angle multiple Coulomb scattering is the cause of the average particle direction inside the radiator differing from the direction as measured by the outside tracking stations. This affects the achievable PID resolution for some particles, as all Cherenkov angles are measured relative to one track. PID is not affected when one can measure Cherenkov photon

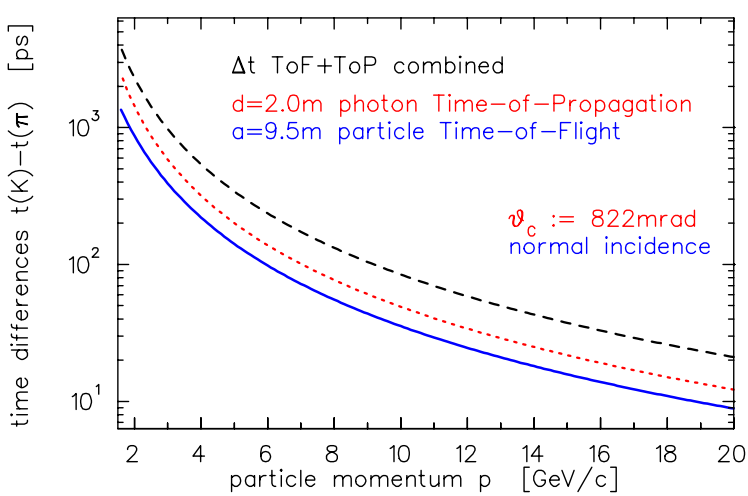

Fig. 4. For one example geometry of particle and photon track, time differences between pion and kaon arrival times for time-of-flight (solid) and time-of-propagation (dotted) components, and the overall photon arrival times (dashed) in TORCH as a function of particle momentum are shown.

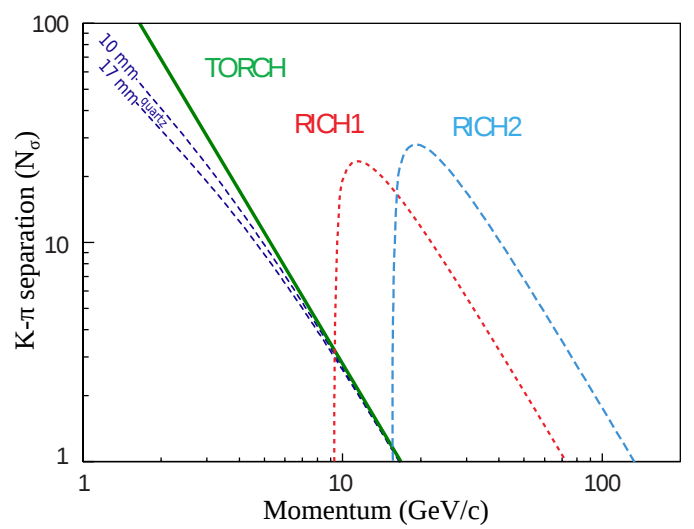

Fig. 5. Calculated performance (in sigma separation) of the different components of the LHCb PID system versus momentum, for isolated tracks (modified from [2]). The dashed blue curves give the effect of multiple Coulomb scattering on performance for quartz plate thicknesses of $10 \mathrm{~mm}$ and $17 \mathrm{~mm}$ (assuming single-sided optical readout and upstream-only tracking).

directions on opposite positions on the Cherenkov cone, and the tracking error cancels. PID is affected when information on the particle direction through the quartz is only available from the nearby tracking stations. In this case, and assuming that the tracking stations located upstream work perfectly, the effect (dashed curves in Fig. 5) on TORCH performance is only sizeable at low momenta where the separation remains much larger than $3 \sigma$.

With a timing resolution of $\sim 15 \mathrm{ps}$ and assuming $\sim 30$ single photons per charged particle, one arrives at a required resolution of $\sim 70 \mathrm{ps}$ per detected photon. This value combines the optical resolution together with the effects of pixelisation on the reconstruction with the resolution of the readout chain of photon detection, amplification, discrimination and digitisation. Both optical and readout resolutions should hence be around $\sim 50 \mathrm{ps}$.

\section{Photon SEnsors}

The design requirements of the MCP to achieve the necessary spatial accuracy are $53 \mathrm{~mm} \times 53 \mathrm{~mm}$ active area with a granularity of $128 \times 8$ (or $64 \times 8$ with charge sharing). The lifetime requirement is that there is no significant degradation up to an integrated collected charge of $5 \mathrm{C} / \mathrm{cm}^{2}$ [8]. 


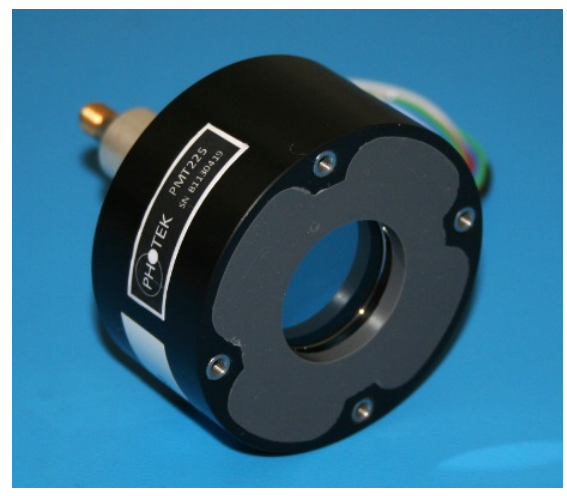

Fig. 6. Front view of the Photek single channel MCP sensor.

The current TORCH design uses custom-made MCPs manufactured by an industrial partner, Photek Limited, UK. For the single channel prototype MCPs (see Fig. 6) Photek has demonstrated lifetimes of better than $5 \mathrm{C} / \mathrm{cm}^{2}$ [9]. With laser tests a time resolution of $\sigma=23 \mathrm{ps}$ has been observed [10].

The first multichannel prototype MCPs have a circular housing $(40 \mathrm{~mm}$ active diameter) with a rectangular active area $(26.5 \times 26.5 \mathrm{~mm})$ and $32 \times 32$ pixels. This geometry represents a quarter-size layout of the active area for the final TORCH MCP design, with comparable pitch density.

\section{Readout Electronics}

To achieve the demanding time resolution of $\sigma=50 \mathrm{ps}$, to instrument the $128 \times 8$ channels per MCP and to fit within the $60 \mathrm{~mm}$ pitch of the MCP photon sensors, customised electronics boards have been developed [11]. For the initial conversion of the MCP signal into a logic time-over-threshold signal, 32-channel NINO chips are used [12]. The HPTDC chip subsequently converts these signals into digital time stamps [13]. By measuring both edges of the time-over-threshold signals, time-walk corrections can be applied to improve measurement uncertainties. By feeding test pulses into two separate channels, a time difference spread of $\sigma=38 \mathrm{ps}$ has been observed which leads to single channel time resolution of $\sigma=38 \mathrm{ps} / \sqrt{2}=26.8 \mathrm{ps}[11]$.

\section{Test Setup}

A scaled-down version of a TORCH module for use in a test beam is currently being assembled, with a $350 \mathrm{~mm} \times 120 \mathrm{~mm} \times 10 \mathrm{~mm}$ quartz radiator plate and a focussing block of matching width (see Fig. 7). The electronics boards are attached in an L-shape configuration so that the beam can reach large areas of the radiator plate unobstructed.

The measured wavelength range is from $600 \mathrm{~nm}$ to well into the UV. Since the Cherenkov angle in the dispersive quartz material is a function of the refractive index, simulations (Fig. 8) show bands of Cherenkov cones instead of narrow lines. Including total internal reflection off the side faces the bands show significant overlap in the left panel. The view in the middle panel shows that these bands are thin twodimensional objects in the three-dimensional space-time and with sufficient spatial and time resolution the bands can be

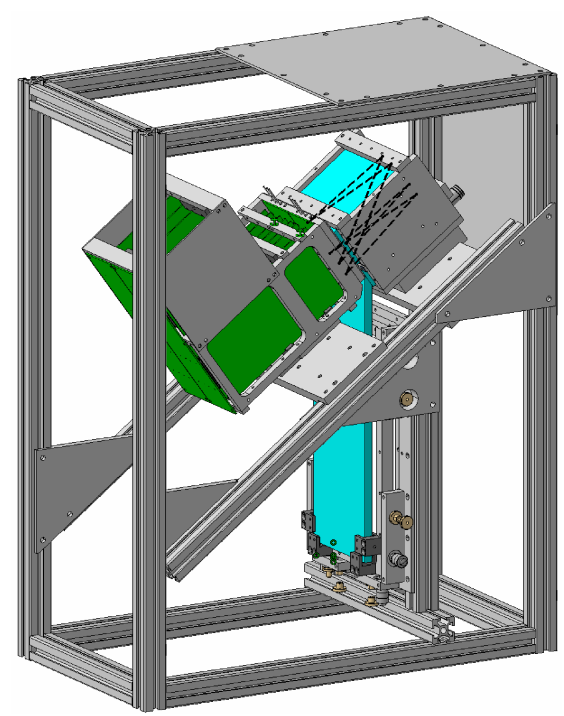

Fig. 7. A drawing of the scaled-down TORCH module for the test beam set-up.

resolved. If the side faces of radiator and focussing block are coated with a black absorbing layer, the number of photons decreases but the pattern being a single band is much easier to analyse (right panel). A method has been developed to reversibly coat quartz surfaces, and it was found that a thin layer of the 2-component transparent silicone PACTAN 8030 (original use is high voltage insulation) loaded with black dye absorbs light well, and that it can easily be peeled off if required.

\section{OPTICS FOR BABAR DIRC COMPONENTS}

After the dismantling of the BaBar experiment at SLAC, the boxes holding the quartz radiator bars of the BaBar DIRC [14] are currently in storage and SLAC has solicited proposals for their possible re-use in other experiments. Each of the 12 planar bar-boxes contains 12 quartz bars $17 \mathrm{~mm} \times 35 \mathrm{~mm} \times 490 \mathrm{~mm}$ in size. The length and the available area of the BaBar bars match the LHCb geometry if they are sited at $9.5 \mathrm{~m}$ distance from the IP.

A wedge attaches at the end of each individual bar (see Fig. 9). The wedge thickness of $33 \mathrm{~mm}$ does not allow to determine the lateral photon angle by measuring particle and photon position as in the baseline TORCH design with its wide radiator plates. On the other hand, the aperture of $33 \mathrm{~mm}$ makes it necessary to focus the photons in the lateral direction as well, as the resolution from the pinhole camera imaging that BaBar used is not sufficient for TORCH.

Figure 9 shows the concept of an optics system to attach to the BaBar DIRC boxes exit window. Spherical surfaces allow focussing in both directions. To keep imaging errors within tolerance, the surface is composed of small slices so that the coarse geometry is quite close to a cylindrical surface. As long as the slice width shown in the figure is not smaller than the quartz bar width (for the case of the object distance being about equal to the imaging distance), the imaging patterns from adjacent mirror slices do not overlap. Separate photon 


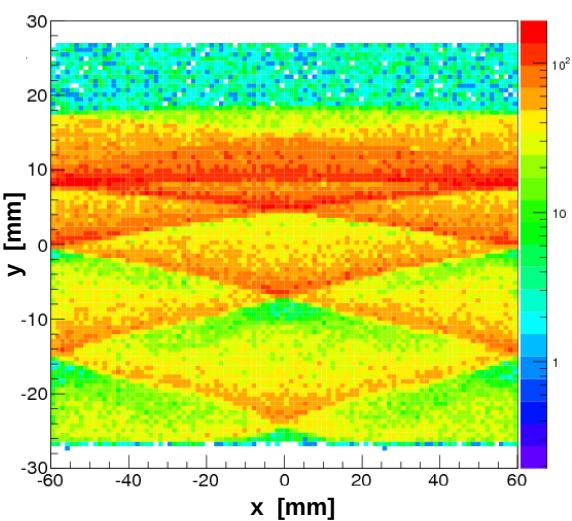

pattern on focal plane

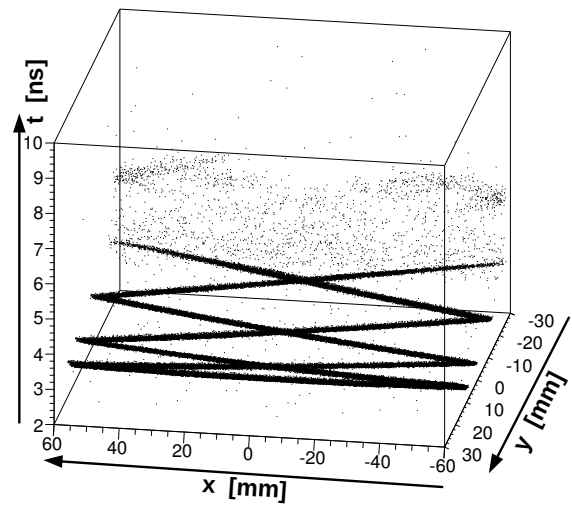

oblique 3-d view including time

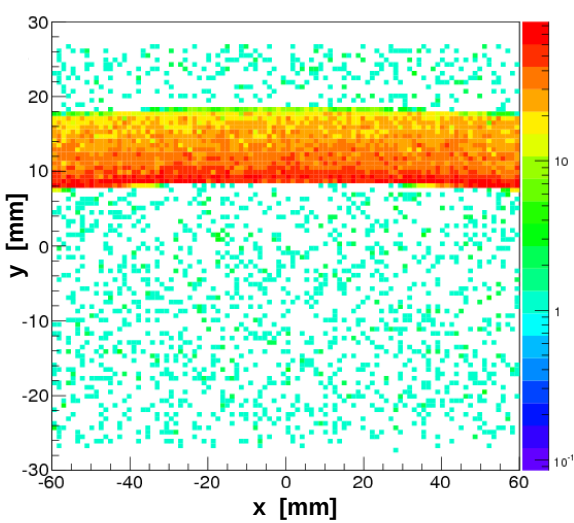

pattern on focal plane

Fig. 8. Simulations for the test beam set-up, running 1000 positive kaons of $10 \mathrm{GeV} / \mathrm{c}$ at $\theta=10^{\circ}$ through the centre of the radiator plate and recording the focal plane hit positions. The left two panels show patterns with the sides of both radiator and focussing block polished, for the right panel these were absorbing (giving a single band). The middle panel expands the view of the left panel with the photon transit time as the third coordinate.

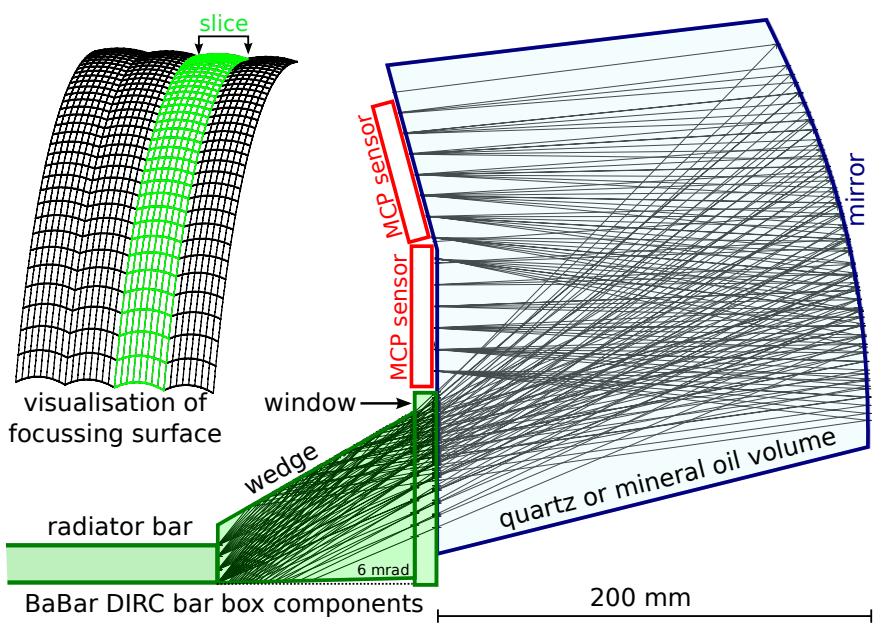

Fig. 9. Conceptual design of an imaging block attaching to the BaBar DIRC box exit window, with the inset shape showing the required focussing surface made out of an arrangement of spherical slices. The green components to the left, end of bar, wedge (upper slope $30^{\circ}$, lower slope $6 \mathrm{mrad}$ upwards from horizontal) and window, are part of the BaBar DIRC bar box.

sensors are used to record the photons that are or are not reflected off the upper wedge slope.

Figure 10 shows the simulated pattern that a Cherenkov cone creates in a glass material without dispersion. The top and bottom curve groups are direct photons and photons reflected off the upper wedge slope, respectively. The resolved double lines in the lower middle are $12 \mathrm{mrad}$ apart, caused by some photons being reflected off the lower wedge slope of $6 \mathrm{mrad}$, and some off the bar bottom. In the middle range ( $\mathrm{x}$ from about $-100 \mathrm{~mm}$ to about $+100 \mathrm{~mm}$ ) the resolution is good enough in both dimensions to achieve the required overall $1 \mathrm{mrad}$ Cherenkov angle resolution.

\section{ViI. Conclusions And Perspectives}

The TORCH detector is designed to provide low-momentum PID in the LHCb experiment. The effect of multiple Coulomb scattering on PID performance has been described. The status

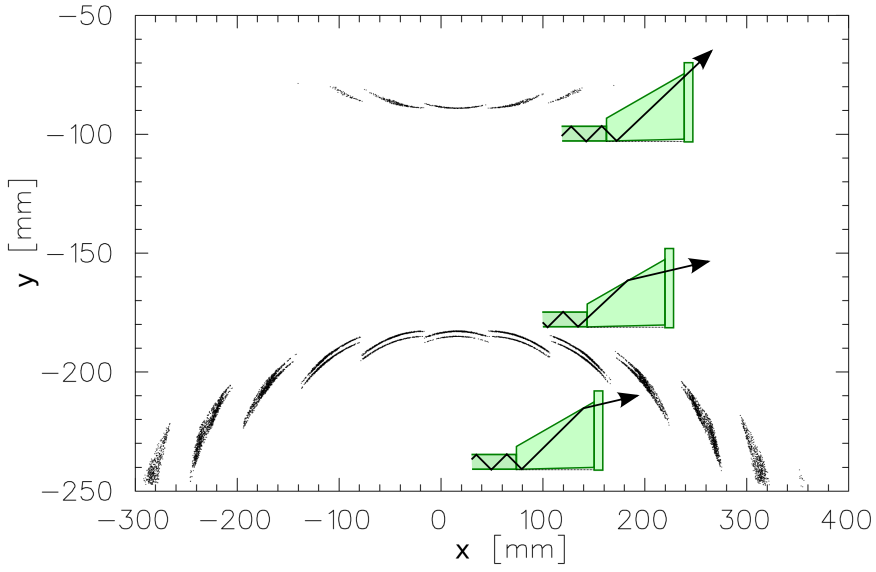

Fig. 10. Cherenkov photon pattern (particle perpendicular to plate, $\beta=1$, $\mathrm{n}=1.47$ ) imaged by an array of spherical surface slices with an effective focal length of $\mathrm{f}=200 \mathrm{~mm}$ and $32 \mathrm{~mm}$ slice width. The insets give the typical photon path through bar and wedge for the three curve groups.

of work on MCP photosensors and electronics and their assembly into a test beam setup has been reported, and supporting Monte Carlo Geant4 simulations presented.

Bar boxes fully equipped with quartz radiators from the dismounted BaBar experiment at SLAC could be used as TORCH modules. Investigations will continue in how they potentially can be used both for large scale prototype work and as building blocks for a TORCH design option.

\section{ACKNOWLEDGMENT}

The support of the European Research Council is gratefully acknowledged in this work (ERC-2011-AdG, 291175TORCH).

\section{REFERENCES}

[1] LHCb Collaboration, The LHCb Detector at the LHC, JINST 3 (2008) S08005, http://dx.doi.org/10.1088/1748-0221/3/08/S08005

[2] LHCb Collaboration, Letter of Intent for the LHCb Upgrade, CERNLHCC-2011-001, LHCC-I-018, http://arxiv.org/abs/arXiv:1310.0183 
[3] M.W.U. van Dijk et al., TORCH - a Cherenkov based time-of-flight detector, NIM A 766 (2014) 118-122, http://dx.doi.org/10.1016/j.nima.2014.04.083

[4] M.J. Charles and R. Forty, TORCH: Time of flight identifcation with Cherenkov radiation, NIM A 639 (2011) 173-176, http://dx.doi.org/10.1016/j.nima.2010.09.021

[5] N. Harnew et al., TORCH: A Large-Area Detector for Precision Timeof-Flight Measurements at LHCb, Physics Procedia 37 (2012) 626-633, http://dx.doi.org/10.1016/j.phpro.2012.02.409

[6] P. Coyle et al., The DIRC counter: a new type of particle identification device for B factories, NIM A 343 (1994) 292299, http://dx.doi.org/10.1016/0168-9002(94)90568-1

[7] LHCb-RICH Collaboration, Upgrade of $\mathrm{LHCb}$-RICH detectors, NIM A 766 (2014) 110-113, http://dx.doi.org/10.1016/j.nima.2014.04.084

[8] J.S. Milnes et al., The TORCH PMT, a close packing, multi-anode, long life MCP-PMT for Cherenkov applications, NIM A 766 (2014) 183-184, http://dx.doi.org/10.1016/j.nima.2014.05.035

[9] T.M. Conneely et al., Extended lifetime MCP-PMTs: Characterisation and lifetime measurements of ALD coated microchannel plates, in a sealed photomultiplier tube, NIM A 732 (2013) 388-391, http://dx.doi.org/10.1016/j.nima.2013.07.023

[10] T. Gys et al., Performance and lifetime of micro-channel plate tubes for the TORCH detector, NIM A 766 (2014) 171-172, http://dx.doi.org/10.1016/j.nima.2014.04.020

[11] R. Gao et al., Development of Scalable Electronics for the TORCH Timeof-Flight Detector, presentation given at TWEPP2014, Aix En Provence, France (22-26 September 2014)

[12] M. Despeisse et al., Multi-Channel Amplifier-Discriminator for Highly Time-Resolved Detection, IEEE TNS Vol. 58, Issue 1 (2011) 202-208, http://dx.doi.org/10.1109/TNS.2010.2100409

[13] M. Mota et al., A flexible multi-channel high-resolution time-to-digital converter ASIC, IEEE Nucl. Sci. Symp. Conf. Rec. 2 (2000) 9/155-9/159, http://dx.doi.org/10.1109/NSSMIC.2000.949889

[14] The BaBar-DIRC Collaboration, The DIRC particle identification system for the BaBar experiment, NIM A 538 (2005) 281357, http://dx.doi.org/10.1016/j.nima.2004.08.129 